A N N A L E S Annales de Bretagne et des Pays de l'Ouest

\title{
Jean-Luc Deuffic (éd.), La Bretagne carolingienne Maurice Carbonnell, Saint Maudez, saint Mandé
}

\section{Bernard Merdrignac}

\section{(2) OpenEdition}

1 Journals

\section{Édition électronique}

URL : http://journals.openedition.org/abpo/517

DOI : $10.4000 / a b p o .517$

ISBN : 978-2-7535-1515-4

ISSN : 2108-6443

\section{Éditeur}

Presses universitaires de Rennes

Édition imprimée

Date de publication : 30 octobre 2009

Pagination : 214-216

ISBN : 978-2-7535-1008-1

ISSN : 0399-0826

\section{Référence électronique}

Bernard Merdrignac, « Jean-Luc Deuffic (éd.), La Bretagne carolingienne

Maurice Carbonnell, Saint Maudez, saint Mandé », Annales de Bretagne et des Pays de l'Ouest [En ligne],

116-3 | 2009, mis en ligne le 30 octobre 2011, consulté le 23 septembre 2020. URL : http://

journals.openedition.org/abpo/517 ; DOI : https://doi.org/10.4000/abpo.517

Ce document a été généré automatiquement le 23 septembre 2020.

(c) Presses universitaires de Rennes 


\title{
Jean-Luc Deuffic (éd.), La Bretagne carolingienne Maurice Carbonnell, Saint Maudez, saint Mandé
}

\author{
Bernard Merdrignac
}

\section{RÉFÉRENCE}

Saint-Denis, Pecia, 2008

Saint-Denis, Jean-Luc Deuffic, 2009

1 Les contributions rassemblées dans le volume 12 de la collection Pecia abordent quelques aspects de l'influence culturelle des monastères dans la diffusion de la Réforme carolingienne en Bretagne durant le haut Moyen Âge. Selon M. Besseyre («Une iconographie sacerdotale du Christ et des évangélistes dans les manuscrits bretons des $\mathrm{IX}^{\mathrm{e}}$ et $\mathrm{X}^{\mathrm{e}}$ siècles $\left."\right)$, les célèbres enluminures anthropozoomorphes des manuscrits liturgiques produits par les scriptoria bretons n'ont guère d'équivalents contemporains hors de la péninsule. L'auteur souligne ici un détail négligé en général par les chercheurs : l'étole dont ces figurations dotent parfois le Christ et les apôtres ! Le Sacramentaire de Gellone (fin du $\mathrm{VIII}^{\mathrm{e}}$ siècle) constitue un précédent unique antérieur d'un siècle aux enluminures bretonnes : Luc y figure avec tête de bœuf, portant l'étole diaconale et présentant l'Évangile ouvert. Ce fait ouvre des perspectives sur la précocité de la réforme carolingienne en Bretagne ainsi que sur la permanence de ces modèles (postérieurs de plus d'un siècle à la décoration du Sacramentaire). Tout en souscrivant à l'hypothèse selon laquelle ce parti iconographique exprime l'insistance sur la mission pastorale des successeurs des apôtres, il est permis de ne pas suivre l'auteur lorsqu'elle reprend (p.25) l'opinion de J.Alexander qui interprétait ces «antiportraits» des évangélistes comme des «œuvres de résistance » au pouvoir 
carolingien (Actes du Colloque du 15e centenaire de l'abbaye de Landévennec,1985). Un beau dossier iconographique rassemblé à la fin de l'ouvrage vient illustrer cette contribution

R. Flechner («Aspects of the Breton transmission of the Hibernensis») relève qu'à la suite de la déposition par Nominoé de cinq évêques de Bretagne accusés de simonie au synode de «Coitlouh » (848-849), la lettre adressée pape Léon IV (846-855) à l'épiscopat breton pour dénoncer cette décision invoque les «sanctorum canonum iudicia » ou les « decretalium regulas » qui se trouvent « aput nos cum illis in canone ». La liste des autorités citées par le pontife permet à l'auteur d'identifier cette documentation canonique avec la Dionysio-Hadriana promulguée par Charlemagne au concile d'Aix-la-Chapelle en 802 dans le but d'unifier la chrétienté. Dès lors, l'auteur applique la même méthode pour identifier les «libelli et commentarii aliorum» auxquels sont censés se référer ses interlocuteurs. Jérôme, Augustin, Isidore et "certeri similiter sancti doctores» sont effectivement les sources de la Collectio canonum Hibernensis, d'origine irlandaise, mais dont la tradition manuscrite prouve qu'elle a été en usage en Bretagne continentale. L'auteur démontre donc qu'elle a revêtu ici une dimension politique essentielle.

3 P.-Y. Lambert (« Exempla bibliques comme précédents judiciaires: les sanctions dans les chartes « celtiques » ) revient sur la Collectio canonum d'origine irlandaise pour y identifier un témoin important de la diffusion d'un type de clause comminatoire qui n'est pas spécifique à la «charte celtique » (telle que définie par W. Davies), puisqu'on le retrouve dans les formulaires mérovingiens et carolingiens, mais que les pays celtiques ont eu tendance à généraliser en la développant (en Irlande et en Bretagne continentale) par des exemples bibliques. Toutefois les formulaires d'excommunication (tels que celui de Lan-Alet = Saint-Germans, Cornwall, sur indication d'Y. Morice à l'auteur) fournissent un parallèle encore plus étroit à ces menaces de sanctions en invoquant les mêmes précédents bibliques: Dathan et Abiron, Saphira et Ananias, Pilate et Judas etc. Les Vitae qui mettent en scène des châtiments analogues (par exemple l'engloutissement vivant du fautif à l'instar de Dathan et Abiron révoltés contre Moïse) démontrent que ces motifs bibliques étaient largement diffusés. À l'appui des conclusions mesurées de l'A, rappelons que la malédiction proférée par Élisée contre Giezi son serviteur indélicat (qui intervient dans les formulaires wisigothiques) se retrouve explicitement dans la Vita Ronani, proférée ici par saint Ronan contre Kéban, son adversaire maléfique.

4 Dans cette même Collectio canonum hibernensis, le cap. 6 qui dénonce la «Britonnum tonsura» est invoqué (p.67, n. 67) par J.T. Mc Ilwain ("The "Celtic» Tonsure Revisited ») pour étayer sa propre hypothèse d'« une tête rasée entièrement, sauf pour une touffe de cheveux sur le front " à l'encontre de l'argumentation de D. Mc Carthy (Celtica, 24 [2003]). Pourtant, la rigoureuse démonstration de ce dernier à l'appui d'une tonsure triangulaire (la base du $\Delta$ à l'arrière du crâne, la pointe sur le devant du front) paraît au recenseur emporter davantage la conviction. Elle est étayée en effet sur des reconstitutions informatisées à partir des enluminures irlandaises; elle a, de plus, l'insigne intérêt de concilier élégamment la description par Ceolfrid de la tonsure d'Adomnán, le témoignage précité de l'Hibernensis et l'allusion de Muirchú à saint Patrice comme " ascicaput » (" tête de hache »).

5 J.-L. Deuffic, éditeur de la collection Pecia, dédie à la mémoire du professeur Gwénaël Le Duc, disparu précocement $(† 2006)$ sa propre contribution intitulée « Le « monachisme breton » continental : ses origines et son intégration au modèle carolingien ». Cet essai de synthèse part de ce que « le monachisme breton n'a guère fait jusqu'à présent l'objet 
d'études générales pertinentes de la part des médiévistes» (p. 77) et se termine sans avoir eu « la prétention d'avoir donné une vision exhaustive de cette période tant est vaste le sujet " (p.137). Bien des titres auxquels se réfère l'impressionnante annotation ont surtout un intérêt historiographique; par contre, on peut être surpris de ne pas retrouver mention de travaux plus récents (sur la lettre de l'épiscopat de la province de Tours aux prêtres bretons Lovocat et Catihern, par exemple - p. 81-83). Le signataire du présent compte rendu ne s'avancerait pas à suivre l'auteur dans certaines affirmations aventurées (ainsi de l'éventuelle participation de titulaires d'abbayesévêchés en Cornouaille et en Domnonée au Concile de Vannes [entre 461 et 491] ou de l'ancienneté d'hypothétiques fondations "celtiques" préexistant aux abbayes SaintMathieu de Finneterre et de Sainte-Croix de Quimperlé). À l'appui d'éventuelles influences de la tradition monastique orientale, on peut aussi trouver particulièrement acrobatique la note qui invoque les relations entre le prieuré Saint-Macaire (à BaguerPican, près de Dol) et l'« ancienne abbaye » de l'île de Saint-Rion, enclave doloise dans l'archipel de Bréhat (p. 85, n. 29) en affirmant que «l'origine [de celle-ci] remonte sans doute au premier monachisme »...

Le culte de saint Rion reste « associé à celui de saint Maudez » ajoute ici l'auteur. C'est l'occasion de signaler la parution, toujours grâce à l'intense activité éditoriale dont il faut féliciter J.-L. Deuffic, de la monographie de M. Carbonnell consacrée à saint Maudez, patron de la ville de Saint-Mandé (94) où réside l'auteur. Fruit d'un patient travail de recherche et de compilation qui a donné lieu à plusieurs publications préliminaires depuis 1997, l'ouvrage fait prudemment le point sur l'historicité de ce saint personnage. Il s'attache surtout à l'extension du culte de saint Maudez: toponymie, anthroponymie, dédicaces, iconographie, spécialisations, manifestations de dévotion, etc. La cinquantaine de pages de cartes, tableaux, photographies en couleurs constituent un modèle de genre et seront fort utiles aux chercheurs intéressés. 